\title{
Lung Age Bio-feedback Using a Portable Lung Age Meter with Brief Advice During Routine Consultations Promote Smoking Cessation - Know2quit Multicenter Randomized Control Trial
}

\author{
Joseph Ojedokun ${ }^{1 *}$, Sarah Keane ${ }^{2}$ and Kieran O'Connor ${ }^{3}$
}

${ }^{1}$ General Practice Specialist Registrar, South East General Practice Training Programme (MBBS; MRCSI; MICGP), Waterford Regional Hospital, Waterford, Ireland ${ }^{2}$ General Practice Specialist Registrar, South East General Practice Training Programme (MB BCh; MICGP), Waterford Regional Hospital, Waterford, Ireland ${ }^{3}$ Senior Medical Officer (MD MPH FRCPI), Department of Public Health, HSE-SE, Kilkenny, Ireland

\begin{abstract}
Background presenting lung age data to smokers who were invited for a formal spirometry has been shown to increase quit rate at 12 months. Main Objective Here, we evaluate the effect of informing active smokers of their lung age using a portable Vitalograph during routine GP consultations on smoking cessation intentions and quit behavior. Methods Four hundred and two active smokers from 5 General Practices had their smoking behaviors and stages on Prochaska's wheel of change (WOC) evaluated before consultation. Patients randomized to the control arm received standardized smoking cessation advice during routine consultations. Patients in the intervention arm received, in addition, lung age information derived from a portable Vitalograph. Self-reported quit rates and progression on the WOC were assessed at 4 weeks post-intervention. Results Quit rates at 4 weeks in the control and intervention arms respectively were $12.0 \%$ and $22.1 \%$ (difference $10.1 \%, p=0.01,95 \% \mathrm{Cl} 1.5 \%$ to $18.7 \%$; number needed to treat 10). Net positive progression on the WOC in the control and intervention arms respectively were $7.3 \%$ and $29.1 \%$ (difference $21.8 \%, p=0.02,95 \% \mathrm{Cl} 13.2 \%$ to $30.4 \%$; number needed to treat 4.6 ). Smokers with poorer lung age values were just as likely to quit as those with normal lung ages. Smokers in the intervention group were more likely to request pharmacotherapy to support quitting $(p<0.0001)$. Conclusion When promoting smoking cessation during clinical consultations, providing 'lung age' biofeedback to patients using a Vitalograph is a clinically effective intervention to foster quitting and positive intentions towards quitting.
\end{abstract}

Keywords: Addiction and abuse (cigarette); General practice/family medicine; Prevention; Health education; Communication; Casecontrol design

\section{Introduction}

Tobacco consumption is unquestionably linked with mortality; it kills up to half of those who smoke regularly [1-3]. As it is difficult to motivate people to quit smoking, new methods to foster quitting are being explored. These include small monetary payments for healthy behavior and providing bio-feedback of spirometrically-determined "lung age", which is the age of an average healthy person with similar spirometric test results [4]. Lung age is commonly estimated from regression equations for the forced expiratory volume in one second (FEV1) in healthy non-smokers, and constitutes the age at which the FEV1 measured in an individual equals the predicted value of FEV1, taking into account age, height, sex and ethnicity $[5,6]$. Quit rate increased in situations where patients were invited for formal spirometry and informed of their lung age as opposed to being given the raw FEV1 value [4,7]. The prime hypothesis of this study was that providing smokers with lung age information during routine consultations using a hand-held lung age meter will foster intentions to quit and actual quit attempts at one month, over and above usual care.

\section{Methods}

\section{Study design}

This is a multicentre randomized controlled trial with an experimental intervention that consists of providing smokers with their lung age information during routine consultations, in addition to usual care. The primary outcome of interest was the proportion of patients abstinent from smoking at one month as a result of the intervention. We considered an incremental quit rate of $10 \%$ as a result of the intervention to be a clinically meaningful effect size in comparison with routine care. On the basis of a one tailed hypothesis that the intervention would not increase smoking behavior, we calculated that a sample size of 250 patients in each study arm would allow us to observe this effect size or one greater with $80 \%$ power at a significance level of $5 \%$. The data were analyzed using SPSS version 18 . The effects of the intervention on nominal and ordinal outcomes were examined using the Chi Square test. The distribution of lung-age across categories of changed smoking behavior was examined using the Kruskal-Wallis test and the Independent Samples Median Test. Intention and readiness to quit smoking was assessed using Prochaska's and DiClemente's transtheoretical Wheel of Change (WOC) model [8]. The relative effects of lung age, Wheel of change and Cessation Support on quit behavior were examined by Ordinal and Logistic Regression. Lung age difference refers to the difference between lung age as determined on the Vitalograph and chronological age.

\section{Questionnaire}

The study questionnaire consisted of sections examining basic demographic data, smoking behaviour including pack years (average

${ }^{*}$ Corresponding author: Joseph Ojedokun, General Practice Specialist Registrar South East General Practice Training Programme (MBBS; MRCSI; MICGP), Waterford Regional Hospital, Waterford, Ireland, Tel: +353 851333 844; E-mail: jojedokun@gmail.com

Received July 27, 2013; Accepted August 22, 2013; Published September 03, 2013

Citation: Ojedokun J, Keane S, O'Connor K (2013) Lung Age Bio-feedback Using a Portable Lung Age Meter with Brief Advice During Routine Consultations Promote Smoking Cessation - Know2quit Multicenter Randomized Control Trial. J Gen Pract 1: 123. doi: 10.4172/2329-9126.1000123

Copyright: (c) 2013 Ojedokun J, et al. This is an open-access article distributed under the terms of the Creative Commons Attribution License, which permits unrestricted use, distribution, and reproduction in any medium, provided the original author and source are credited. 
Citation: Ojedokun J, Keane S, O'Connor K (2013) Lung Age Bio-feedback Using a Portable Lung Age Meter with Brief Advice During Routine Consultations Promote Smoking Cessation - Know2quit Multicenter Randomized Control Trial. J Gen Pract 1: 123. doi: 10.4172/23299126.1000123

Page 2 of 5

number of cigarettes smoked per day multiplied by the number of years smoked divided by 20), the stage on the wheel of change for intention to quit, known co-morbidities and documentation of the type of cessation support provided during the initial consultation. Information on previous quitting (number of attempts and types of cessation support required), smoking status of partners and current employment status were used to confirm baseline comparability of groups. At one month after the initial consultation, a second questionnaire was sent to all participants. They were examined for changed smoking behavioral patterns and intentions to quit on the wheel of change (Table 1). Telephone calls were made to patients after a further 2 weeks if questionnaires were not returned to maximize response rate.

\section{Outcome measures}

\section{Primary:}

- Rates of change of smoking behavior at one month, with possible categories of (i) quit for one month, (ii) quit for the previous week, (iii) stopped smoking, but relapsed, (iv) smoking the same number of cigarettes, (v) smoking more. Abstinence for one month was considered the most salient outcome; the effect size and the number needed to treat are based on this criterion.

- Stage on the wheel of change as regards intention to stop smoking, with possible categories of (i) pre-contemplation, (ii) contemplation, (iii) preparation (iv) action (Table 1).

\section{Secondary:}

- Effects of lung age difference and stage on the wheel of change on quit outcomes.

\section{Study protocols}

Protocols were standardized across all investigating General Practices and included:

- Guidelines on the delivery of smoking cessation advice in a personalized fashion based on the A, B, and C guidelines of the ICGP and New Zealand guidelines [9].

- Vitalograph - Detailed instructions on the use of the COPD-6 Vitalograph.

- Pharmacotherapy - prescription guidelines for Varenicline, Bupropion and Nicotine Replacement Therapy based on the British National Formulary (BNF 63).

- Patient information leaflet - all participants received the same type of advice leaflet - just be smoke free - from the Irish Cancer Society [10].

\begin{tabular}{|l|l|}
\hline \multicolumn{1}{|c|}{ Stage } & \multicolumn{1}{c|}{ Description } \\
\hline Precontemplation & $\begin{array}{l}\text { I am not thinking about quitting. I am not ready to make } \\
\text { any changes to my smoking right now. }\end{array}$ \\
\hline Contemplation & $\begin{array}{l}\text { I am beginning to think about stopping smoking. I am } \\
\text { weighing up the positives and negatives of quitting. }\end{array}$ \\
\hline Preparation & $\begin{array}{l}\text { I have decided to try to quit smoking. I am starting to look } \\
\text { at why I am smoking. I am trying to reduce the amount } \\
\text { I smoke and trying to figure out the best way to start } \\
\text { stopping. }\end{array}$ \\
\hline Action* & $\begin{array}{l}\text { Answers 'Yes' to 'I have not smoked cigarette in the last } \\
\text { one month'. }\end{array}$ \\
\hline
\end{tabular}

*The action phase was assessed in the follow-up questionnaire.

Table 1: Stages of the Wheel of Change - Questionnaire Assessment (initial and follow-up).

\section{Randomization procedure}

Allocation groups, as determined by computer-generated random numbers (even=control), were concealed in sequentially numbered opaque sealed envelopes by an independent clerk unrelated to the Practices.

\section{Recruitment, allocation and intervention}

All patients presenting to an investigating GP on a given day regardless of the reasons for routine consultations were presented with a research pack at the desk. Non-smokers indicated their status on the front page of the pack and were excluded. From the pack, all smokers received study information and were invited to consent to participation if they did not meet the exclusion criteria (Figure 1). These included unavailability for follow-up, enrolment in another smoking cessation research study, current use of smoking cessation pharmacotherapy, use of domiciliary Oxygen, history of major lung disease (lung cancer, Tuberculosis, Sarcoidosis) and cognitive dysfunction. Typically, questionnaires were completed in the waiting rooms.

At an appropriate stage during the consultation, the doctor opened the next of serially numbered opaque sealed envelopes to reveal the allocation group for each patient. All patients received standardized personalized brief smoking cessation advice including an offer of cessation support in the form of pharmacotherapy or a follow-up review as appropriate and also the standard patient information leaflet. Participants in the intervention arm additionally had their lung age assessed using the desktop Vitalograph lung age meter. Lung age results were explained, recorded on an advice slip and given to these patients.

\section{The vitalograph}

The Vitalograph lung age meter is a portable desktop device, which has been validated to evaluate the actual and \% predicted FEV1 to an accuracy better than $\pm 3 \%$ (performance standard: American Thoracic Society and European Respiratory Standards 2005) [11]. It estimates the lung age to help illustrate the impact of smoking on the subject's lungs based on the age, height, gender and FEV1.

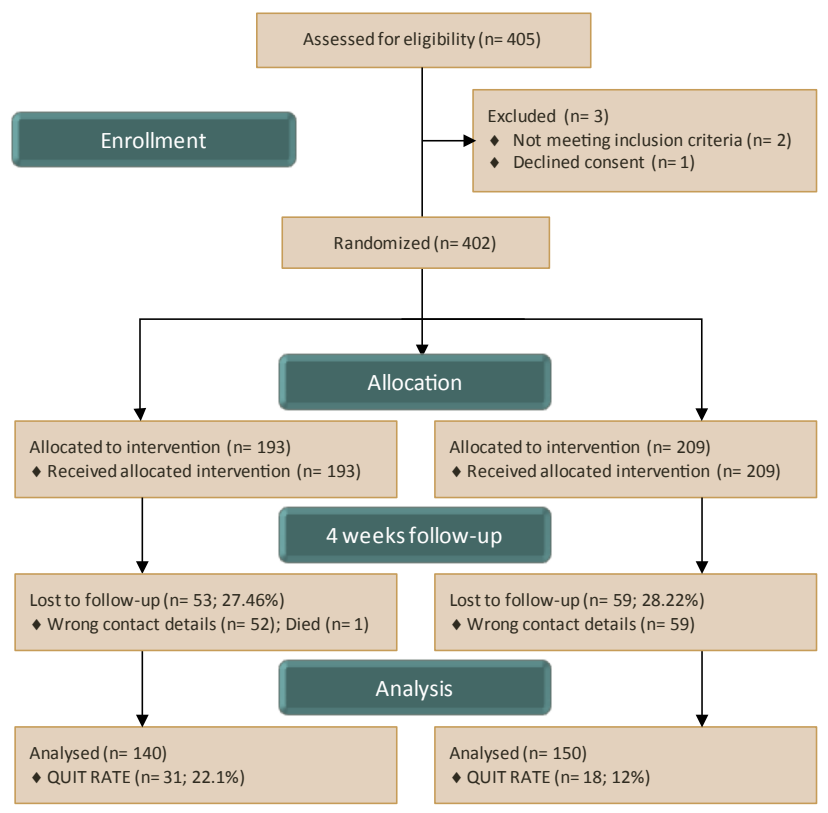

Figure 1: Flow diagram of the randomized control trial. 
Citation: Ojedokun J, Keane S, O'Connor K (2013) Lung Age Bio-feedback Using a Portable Lung Age Meter with Brief Advice During Routine Consultations Promote Smoking Cessation - Know2quit Multicenter Randomized Control Trial. J Gen Pract 1: 123. doi: 10.4172/23299126.1000123

Page 3 of 5

\section{Settings}

Two rural and 3 urban General Practices located in the SouthEast of Ireland. Patients were recruited to the study over a period of 6 months.

\section{Results}

\section{Baseline characteristics}

The mean age of all patients was 39.7 years with a standard deviation (SD) of 14.8 years. The intervention arm did not differ from the controls ( $p>0.05)$ with respect to all measured baseline characteristics (Table 2). Lung ages were skewed towards higher values; mean, median and standard deviation values were $17.5,14.0$ and 18.7 years respectively.

\section{Follow-up - quit rates}

Self-reported quit rates at 4 weeks in the intervention and control arms respectively were $22.1 \%$ and $12 \%$ (difference $10.1 \%$, Chi square $=14.9 ; \mathrm{df}=5 ; \mathrm{p}=0.01 ; 95 \% \mathrm{CI} 1.5 \%$ to $18.7 \%$; number needed to treat 10$)$. By contrast, $12.1 \%$ of smokers in the intervention arm versus $4.7 \%$ controls had relapsed within one month (Figure 2). Fewer patients in the intervention group, $39.9 \%$ versus $46.7 \%$ in controls, were smoking the same number of cigarettes after 4 weeks. Within the intervention group, lung age did not differ significantly across all possible categories of quit outcomes $(\mathrm{p}=0.88)$, indicating that smokers

\begin{tabular}{|c|c|c|c|}
\hline Variable & $\begin{array}{l}\text { Control }(n=209) \\
\text { Mean; Median; SD }\end{array}$ & $\begin{array}{l}\text { Intervention (n } \\
\text { = 193) Mean; } \\
\text { Median; SD }\end{array}$ & $\begin{array}{l}\text { Intervention (n } \\
=193 \text { ) Mean; } \\
\text { Median; SD }\end{array}$ \\
\hline $\begin{array}{l}\text { Age - mean; } \\
\text { median; SD }\end{array}$ & $40.8 ; 40.0 ; 14.9$ & $38.5 ; 37.0 ; 14.6$ & 0.113 \\
\hline $\begin{array}{c}\text { Pack years - } \\
\text { mean; median; SD }\end{array}$ & $19.0 ; 15.0 ; 17.0$ & $16.6 ; 12.0 ; 16.1$ & 0.139 \\
\hline Males (\%) & $80(38.3)$ & $78(40.4)$ & 0.661 \\
\hline Actively employed & $95(45.5)$ & $71(36.8)$ & 0.78 \\
\hline $\begin{array}{l}\text { Socio-economic } \\
\text { groups: }\end{array}$ & & & 0.33 \\
\hline - Professional & $8(3.8)$ & $4(2.1)$ & \\
\hline - Self-employed & $15(7.2)$ & $13(6.7)$ & \\
\hline - Clerical & $27(13)$ & $18(9.3)$ & \\
\hline - Skilled manual & $35(16.7)$ & $24(12.4)$ & \\
\hline - Semi-skilled & $9(4.3)$ & $5(2.6)$ & \\
\hline - Unskilled & $112(53.6)$ & $127(65.8)$ & \\
\hline Level of education: & & & 0.59 \\
\hline - Primary & $39(18.7)$ & $31(16.1)$ & \\
\hline - Secondary & $124(59.3)$ & $122(63.2)$ & \\
\hline - Tertiary & $46(22)$ & $39(20.2)$ & \\
\hline Co-morbidities & & & 0.07 \\
\hline Partner smokes & $97(46.1)$ & $87(45.1)$ & 0.79 \\
\hline $\begin{array}{c}\text { Previously } \\
\text { attempts at quitting }\end{array}$ & & & 0.75 \\
\hline - Never attempted & $35(16.7)$ & $34(17.6)$ & \\
\hline - Attempted once & $63(30.1)$ & $59(30.6)$ & \\
\hline $\begin{array}{c}\text { - More than one } \\
\text { attempt }\end{array}$ & $111(53.1)$ & $99(51.3)$ & \\
\hline Stage on the WOC & & & 0.89 \\
\hline $\begin{array}{c}\text { • Pre- } \\
\text { contemplative }\end{array}$ & $44(21.1)$ & $38(19.7)$ & \\
\hline - Contemplative & $84(40.2)$ & $82(42.5)$ & \\
\hline - Preparation & $81(38.8)$ & $73(38.8)$ & \\
\hline
\end{tabular}

SD: Standard Deviation; WOC: Wheel of Change

Table 2: Baseline characteristics of groups. Figures are absolute numerical values unless stated otherwise.

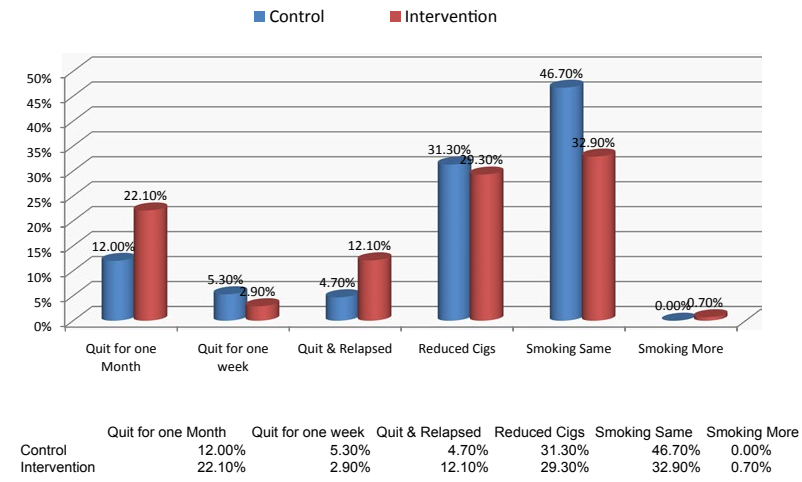

Figure 2: The effect of lung age feedback on quit outcomes.

with poorer lung age values were just as likely to quit as those with normal lung ages.

\section{Progression on the wheel of change}

The net positive progressions on the wheel of change in the control and intervention arms respectively were $7.3 \%$ and $29.1 \%$ (difference $21.8 \% ; \mathrm{Chi}=14.9 ; \mathrm{df}=6 ; \mathrm{p}=0.02,95 \% \mathrm{CI} 13.2 \%$ to $30.4 \%$; number needed to treat 4.6) (Figure 3).

In the bivariate analysis, stage on the wheel of change was a strong predictor of smoking cessation, with smokers in the action stage being much more likely to quit than those in pre-contemplation, (Chi 22.1; df $10 ; \mathrm{p}<0.05)$. However in the multivariate analysis, there was no residual effect on quitting of stage on the wheel of change, after accounting for the independent effect of providing smokers with information on their lung age (action, $\mathrm{p}=76$; contemplation, $\mathrm{p}=0.08$; pre-contemplation $\mathrm{p}=0.11$; lung age feedback $\mathrm{p}<0.05$ ).

\section{Effect of lung age versus cessation support on quitting}

Smokers in the intervention group were more likely than controls to request pharmacotherapy (Chi 25.0; $\mathrm{df} 6 ; \mathrm{p}<0.0001$ ). A multivariate analysis showed that lung age information and cessation support interventions had independent but significant $(\mathrm{p}<0.05$ and $\mathrm{p}<0.001$ respectively) effects on promoting cessation.

\section{Discussion}

Only one patient decline consent to participate in this study indicating that, this was a well-motivated study population who did not view participating in research as a burden. The relatively young mean age (39.7 years) of all participants is consistent with data from the Irish SLÁN report and indicates that smokers in this study are representative of the age cohort of the Irish population in which smoking is most prevalent [12]. The median difference between lung age and chronological age in this group of smokers was 14 years, confirming the harm to lung function caused by continued smoking. Hence, we advocate that practitioners take the opportunity at all consultations to promote smoking cessation interventions.

Our principal finding is that, in addition to brief cessation support during routine consultations, providing lung age bio-feedback to smokers along with pharmacotherapy significantly increases the proportion who quit within a month. One in 10 smokers will quit with this method. Although previous studies did show a clinically significant effect of lung age on quitting using formal spirometry, this is the first study that assessed a similar effect using the portable Vitalograph lung 


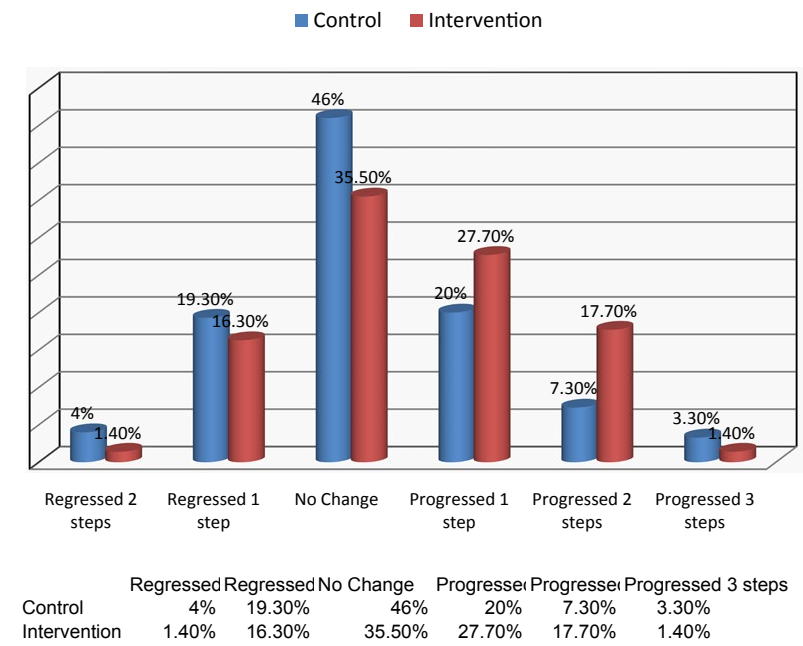

Figure 3: The effect of lung age feedback on intention to quit (progression on the wheel of change).

age meter during routine consultations. Spirometry will screen smokers for chronic lung diseases, but it is neither practical nor cost-effective to use in primary care to encourage smoking cessation. Indeed, in a previous survey, only a little over half (53.7\%) of Irish GPs had access to spirometry within their own Practices [13]. Compared to a spirometer, the Vitalograph is much cheaper, easily accessible and requires no additional training for use.

The lack of biochemical validation for self-reported smoking cessation rates is a limitation of this study. The desirability of biochemical validation versus self-report indices of quitting has been studied previously $[14,15]$. A review conducted by the Society for Research on Nicotine and Tobacco to assess the utility of biomarkers suggested that in large-population, low-intensity trials, biochemical verification was neither feasible nor necessary [16]. The review further suggested that, while it is likely that quit rates are inflated when using self-reports, the amount of inflation is small. Patrick et al. and other large studies have found generally high levels of accuracy, specificity and sensitivity for self-report [17]. These findings suggest that self-reporting may be a reasonable practical indicator of smoking status among smokers that attend their family physicians for routine consultations.

The fact that a significantly higher proportion of smokers who were told their lung age (12.1\% intervention versus $4.7 \%$ control) during this brief intervention had relapsed within one month indicates that although providing lung age bio-feedback prompts initial quitting, more support is required to keep smokers abstinent. Many smokers quit successfully after a few attempts and studies have shown that the more attempts smokers make to quit, the more they are likely to finally succeed [18]. The smokers who relapse may represent a malleable group that might benefit from intensive targeted multimodal interventions including counseling, motivational interviewing, contingency contracting and pharmacotherapy.

We found that within the intervention group, smokers with more advanced lung ages were just as likely to quit as those with lung ages closer to their chronological age. It is important to demonstrate that the effect of lung age biofeedback to foster quitting is not confined primarily to smokers with advanced lung disease, as these smokers would reap less benefit from the intervention.

We further observed that providing lung age feedback to smokers is useful in fostering intentions to quit. Although smokers in both groups were closely matched on the wheel of change at baseline, we demonstrated that those smokers who received the biofeedback intervention had greater forward progression on the wheel of change from pre-contemplation, through contemplation to the action stage. In so far as intention may predict future changed behavior, this is encouraging, as this study was of a short duration and therefore unable to measure long-term progression to maintenance and transcendence from smoking.

Consistent with previous studies, our results also demonstrate that informing smokers of their lung age is equally effective across all stages of intention to quit. This implies that the intervention may also be effective in those hard-to-reach smokers who were not considering quitting prior to the consultation. All smokers, regardless of the level of preparedness to quit, should be encouraged to give up smoking using the Vitalograph bio-feedback and supplemental information and interventions. If more smokers had been recruited, it is possible that an interaction might be observable at the contemplation stage, whereby smokers at this stage would be more amenable to quitting when provided with their lung age. There may be a problem with type 2 error in that our study was insufficiently powered to examine quit effects in multiple subgroups. Nonetheless, it is important to note that providing lung age information may be valuable irrespective of baseline intention to quit.

Traditionally, attrition rates are relatively high in smoking cessation studies. For example, Murray et al. (1983) and Biglan et al. (1985) reported rates as high as $28.9 \%$ in smoking cessation studies depending on the length of the follow-up period. We observed a similar attrition rate, approximately $27 \%$ in both study arms. Most patients were lost to follow-up due to wrong contact details. Anecdotally, some patients who presented later after the study confirmed that their contact details (addresses and phone numbers) had changed and these were not updated in the GP software database. This should be borne in mind and measures put in place to minimize such an error in future studies. Our follow-up time was four weeks. A longer follow-up period would have allowed us to better evaluate the durability of telling smokers their lung age on quitting. Subsequent consultations with some patients who received the intervention revealed that many actually quit smoking after the 4-week follow-up period.

The methodology of this study reduces the potential for invitation bias or the motivated-volunteer effect as our cohort represents smokers who were 'caught in the very act' in that they had sought the consultation for other reasons and not specifically with a view to seeking smoking cessation support. With respect to age, gender, co-morbidities, smoking history, socio-economic status and preparedness to change smoking behavior, our study cohort are representative of typical smokers a GP might encounter on a regular basis regardless of practice type and style. Consequently our study has good generalizability. Although assessing lung age does add an extra 1 to 2 minutes to the consultation time, this can translate into a cost-effective and clinically effective intervention, which can be easily incorporated into routine consultations.

\section{Conflict of Interest}

Authors disclose no conflicts of interests or affiliation with any organization, or company that might bias this work.

\section{Funding}

Funding was provided through a research grant from the Research and education Foundation of the Irish College of General Practitioners. 
Citation: Ojedokun J, Keane S, O'Connor K (2013) Lung Age Bio-feedback Using a Portable Lung Age Meter with Brief Advice During Routine Consultations Promote Smoking Cessation - Know2quit Multicenter Randomized Control Trial. J Gen Pract 1: 123. doi: 10.4172/23299126.1000123

Page 5 of 5

\section{Acknowledgement}

- GP investigators and Support Staff:

o Dr Mark Walsh - Northgate Medical Centre, New Ross, County Wexford. o Dr Karl Roulston - Northgate Medical Centre, New Ross, County Wexford. o Dr Niamh Carew - Northgate Medical Centre, New Ross, County Wexford. o Dr Shane Kavanagh - Whiterock Family Practice, County Wexford. o Dr Jane Ojedokun - Parnell Clinic, Waterford City, Waterford.

- Dr Martin Rouse - Research coordinator/Programme Director, South East GP Training Programme.

\section{References}

1. WHO Report on Global Tobacco Epidemic (2008).

2. Doll R, Peto R, Wheatley K, GrayR, Sutherland I (1994) Mortality in relation to smoking: 40 years' observations on male British doctors. BMJ 309: 901-911.

3. Doll R, Peto R, Boreham J, Sutherland I (2004) Mortality in relation to smoking: 50 years' observations on male British doctors. BMJ 328: 1519.

4. Grossman E, Sherman S (2008) Telling smokers their "lung age" promoted successful smoking cessation. Evid Based Med 13: 104.

5. Morris JF, Temple W (1985) Spirometric "lung age" estimation for motivating smoking cessation. Prev Med 14: 655-662.

6. Toda R, Hoshino T, Kawayama T, Imaoka H, Sakazaki Y, et al. (2009) Validation of "lung age" measured by spirometry and handy electronic FEV1/ FEV6 meter in pulmonary diseases. Intern Med 48: 513-521.

7. Parkes G, Greenhalgh T, Griffin M, Dent R (2008) Effect on smoking quit rate of telling patients their lung age: the Step2quit randomised controlled trial. BMJ 336: 598-600.
8. Prochaska JO, DiClemente CC (1983) Stages and processes of self-change of smoking: toward an integrative model of change. J Consult Clin Psychol 51: 390-395.

9. Ministry of Health (2007) New Zealand smoking cessation guidelines. Ministry of Health, Wellington.

10. http://www.cancer.ie/pdfs/HP_Smoke_free.pdf

11. http://www.vitalograph.ie/downloads/brochures/14499.pdf

12. Slan 2007 (2009) Smoking Patters in Ireland: Implications for Policies \& Services. Dept of Health \& Children, Ireland.

13. National Irish COPD (Respiratory) Strategy 2008. (2007) Survey of General Practitioners on services for people with COPD: Communication - Irish College of General Practitioners.

14. Velicer WF, Prochaska JO, Rossi JS, Snow MG (1992) Assessing outcome in smoking cessation studies. Psychol Bull 111: 23-41.

15. Connor Gorber S, Schofield-Hurwitz S, Hardt J, Levasseur G, Tremblay M (2009) The accuracy of self-reported smoking: a systematic review of the relationship between self-reported and cotinine-assessed smoking status. Nicotine Tob Res 11: 12-24.

16. Benowitz NL, Jacob III P, Ahijevych K, Jarvis MJ, Hall S, et al. (2002) Biochemical verification of tobacco use and cessation. Nicotine Tob Res 4 149-159.

17. Yeager DS, Krosnick JA (2010) The validity of self-reported nicotine product use in the 2001-2008 National Health and Nutrition Examination Survey. Med Care 48: 1128-1132.

18. Hughes JR (2000) New treatments for smoking cessation. CA Cancer J Clin 50: $143-151$. 